\title{
Resolution in Carrier Profiling Semiconductors by Scanning Spreading Resistance Microscopy and Scanning Frequency Comb Microscopy
}

\author{
Mark J. Hagmann*, Marwan S. Mousa ${ }^{1}$, Dmitry A. Yarotski ${ }^{2}$ \\ NewPath Research L.L.C., Salt Lake City, UT 84115, USA \\ ${ }^{1}$ Department of Physics, Mu'tah University, Al-Karak 61710, Jordan \\ ${ }^{2}$ Center for Integrated Nanotechnologies, Materials Physics and Applications Division, \\ Los Alamos National Laboratory, Los Alamos, NM 87545, USA
}

*Correspondence to:

Hagmann MJ,

Tel: +1-801-573-9853

Fax: +1-801-581-5281

E-mail: newpathresearch@gmail.com

Received August 1, 2017

Revised August 15, 2017

Accepted August 15, 2017

\begin{abstract}
High resolution measurements of the carrier profile in semiconductor devices is required as the semiconductor industry progresses from the $10-\mathrm{nm}$ lithography node to 7 -nm and beyond. We examine the factors which determine the resolution of the present method of scanning spreading resistance microscopy as well as such factors for the newer method of scanning frequency comb microscopy that is now under development. Also, for the first time, we consider the sensitivity of both methods to the location of heterogeneities in the semiconductor. In addition, mesoscopic effects on these measurements are considered for the first time. Two simple analytical models are extended to study the sensitivity to heterogeneities as well as mesoscopic effects.
\end{abstract}

Key Words: Carrier profiling, Scanning spreading resistance microscopy, Scanning frequency comb microscopy

\section{INTRODUCTION}

The semiconductor industry is progressing to finer lithography nodes, approximately doubling the number of components per chip each year in accordance with Moore's Law (Mack, 2011) to meet the demand for higher performance. However, as the chip capacity in flash memory increased by 100 -fold from 2005 to 2013 the number of reliable state changes decreased at each step to a finer lithography so the reliable capacity "has actually stagnated" (Chien \& Karamcheti, 2013). Others emphasize the need for higher resolution in lithography at the finer nodes (Rathod et al., 2014) but we address the additional requirement for finer resolution in metrology to better characterize these devices. Semiconductor metrology requires both dopant profiling and carrier profiling to determine the number of dopant atoms and carriers per unit volume. These two procedures are generally used together because dopant profiling verifies that the design was followed and carrier profiling validates the dopant profile and verifies the activation of the dopant atoms. Atom probe tomography provides sub-nm resolution in dopant profiling (Kelly \& Miller, 2007) and we consider new technology to complement this with finer resolution in carrier profiling (Hagmann et al., 2015). The present method of scanning spreading resistance microscopy (SSRM) as well as our new technology of scanning frequency comb microscopy (SFCM) are defined in the following two paragraphs.

SSRM is now the preferred method for carrier profiling at and below the 20-nm lithography node. In SSRM an accuratelycontrolled pressure, typically $15 \mathrm{GPa}$, is required to insert a probe of doped conductive diamond into a semiconductor to measure the spreading resistance at this contact (Hantschel et al., 2015). Comparison with previous measurements, made using this probe with the same applied direct current (DC) bias with standards having known concentrations of the same dopant, are interpolated to evaluate the local carrier density in

(a) This is an open-access article distributed under the terms of the Creative Commons Attribution Non-Commercial License (http://creativecommons.org/licenses/by-nc/4.0) which permits unrestricted noncommercial use, distribution, and reproduction in any medium, provided the original work is properly cited.

Copyrights () 2017 by Korean Society of Microscopy 


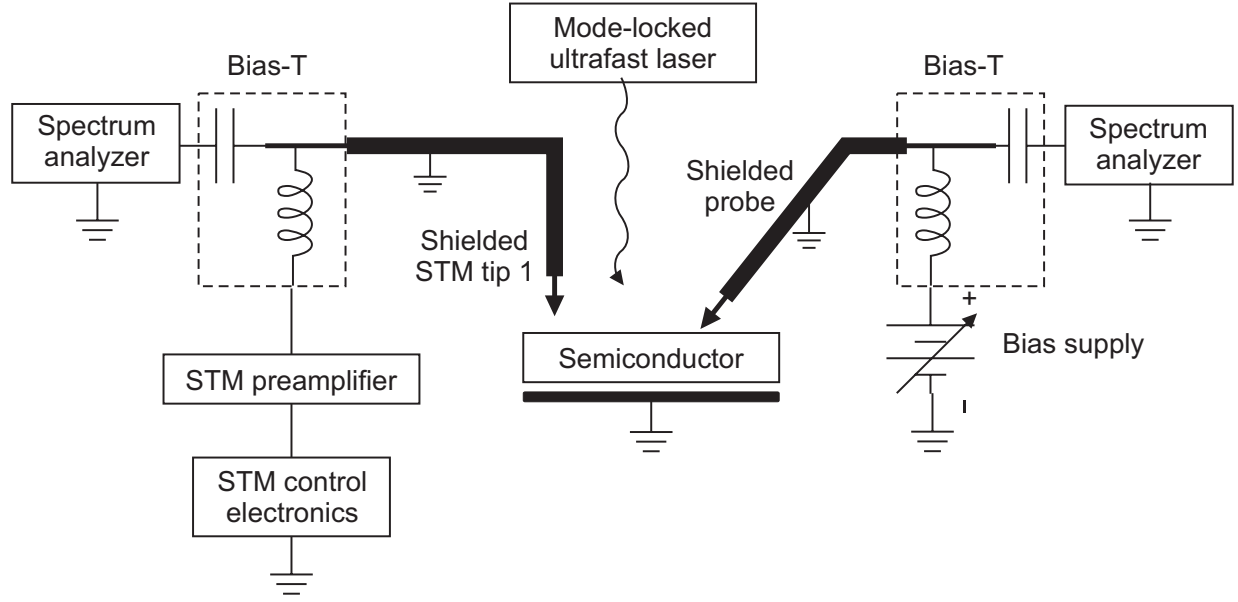

Fig. 1. Block diagram of apparatus for scanning frequency comb microscopy. the sample. Fragile three-dimensional (3D) structures such as Fin-FETs which are used at the finer lithography nodes must be disassembled, polished, and sectioned before SSRM studies (Vandervorst et al., 2014) because they would be destroyed by the high applied pressure. The diamond-probes must be normal to the sample surface to prevent sliding during their high-pressure insertion into a semiconductor which also complicates the testing of intact 3D structures.

In SFCM the semiconductor is the sample electrode in a scanning tunneling microscope (STM) (Hagmann et al., 2017). A mode-locked laser is focused on the STM tunneling junction to generate microwave harmonics at integer multiples of the laser pulse repetition rate (Hagmann et al., 2012) and the attenuation of each harmonic is measured as it propagates from the tunneling junction through the spreading resistance. Comparison of this attenuation with previous measurements made with standards is used to evaluate the local carrier density in the semiconductor. Fig. 1 is a block diagram of the apparatus for SFCM. The power in the microwave frequency comb is measured by two spectrum analyzers, one in the STM tip circuit and the other at a surface probe near the tunneling junction, to determine the attenuation that is caused by the spreading resistance.

Three recent publications claim "nanometer" or "subnanometer" resolution in carrier profiling with SSRM (Hantschel et al., 2009, 2015, 2016). Scanning electron microscope (SEM) images show that the initial apex radius of the diamond probes may be as small as 10 15 nm (Hantschel et al., 2016) or $50 \mathrm{~nm}$ (Hantschel et al., 2015) before scanning. However, an SEM image taken "after a few hours" of scanning shows that by then the apex has a radius of $300 \mathrm{~nm}$ and silicon debris "shaved-off" from the semiconductor is attached to the probe as far as $300 \mathrm{~nm}$ from the apex (Hantschel et al., 2015). Thus, SSRM effectively averages the spreading resistance over a contact that has a radius between $0 \mathrm{~nm}$ and $50 \mathrm{~nm}$ initially and approximately $300 \mathrm{~nm}$ later in the measurements.
Deconvolution is not used to correct for the effects of this averaging, but rather the resolution is generally defined as the minimum lateral displacement of the probe that is required to cause a significant change in the measured resistance. Others have studied the effect of piecewise-homogeneous layered semiconductor samples on SSRM (Dunham et al., 1994) but we see no treatment of the effects of general inhomogeneities or of the possibility of mesoscopic effects in these measurements.

\section{MATERIALS AND METHODS}

It is useful to define the effective radius or "spot-size" as the radius of the contact of the diamond probe with the semiconductor in SSRM, and the radius of a circle subtending one-half of the total tunneling current in SFCM. In SSRM the effective radius is typically from 10 to $300 \mathrm{~nm}$ as noted in the previous paragraph. However, In SFCM the effective radius is typically less than $1 \mathrm{~nm}$ depending on the shape and size of the tip electrode and its distance from the sample (Hagmann \& Henage, 2016). Now we consider several models to address how spot-size and mesoscopic effects determine the sensitivity of SFCM and SSRM to the local inhomogeneities in a semiconductor.

\section{First Model: Hemispherical Metallic Protrusion into the Surface of the Semiconductor}

If a metal sphere with radius a is at the center of a metallic shell having radius $\mathrm{R}$, and the medium between them has constant resistivity $\rho$, the resistance between these two metal surfaces is given by

$$
R_{S}=\frac{\rho}{4 \pi a}\left(1-\frac{a}{R}\right)
$$

Thus, in SSRM if we approximate the contact between the inserted probe and the semiconductor as a hemisphere the 
measured resistance is given by the following expression:

$$
R_{H S}=\frac{\rho}{2 \pi a}\left(1-\frac{a}{R}\right)
$$

In the limit as $\mathrm{R}$ becomes large $\mathrm{R}_{\mathrm{HS}}$ approaches $\rho / 2 \pi \mathrm{a}$. Since the surfaces of constant potential are spheres it may be shown that $1 / 2$ of this total resistance is over the radial distance from a to $2 \mathrm{a}, 1 / 6$ is from $2 \mathrm{a}$ to $3 \mathrm{a}, 1 / 12$ is from $3 \mathrm{a}$ to $4 \mathrm{a}$, and $1 / 20$ is from $4 \mathrm{a}$ to $5 \mathrm{a}$ as shown in Fig. 2. Thus, $80 \%$ of the total resistance is contained within a distance of $4 \mathrm{a}$ from the surface of the central sphere, and $90 \%$ within a distance of $9 a$ from this surface.

Next we will examine the sensitivity of a resistance measurement to the distance R' from the origin which is at the center of the metal sphere. Consider a sphere with radius $\mathrm{R}^{\prime}$ in spherical polar coordinates $r, \theta$, and $\varphi$, so that the volume of the sector bounded by the cone $\theta=\theta_{0}$ is given by

$$
V_{S}=\frac{2 \pi R^{\prime 3}}{3}\left[1-\cos \left(\theta_{0}\right)\right] \approx \frac{\pi R^{\prime 3}}{3} \theta_{0}^{2} \text { for } \theta_{0}<<1 .
$$

Thus, the volume of a short segment of the sector that is bounded by $\mathrm{R}^{\prime}$ and $\mathrm{R}^{\prime}+\Delta \mathrm{R}$ is given by

$$
\Delta V=\pi \theta_{0}^{2} R^{2} \Delta r
$$

The area subtended by this segment is $A=\pi R^{\prime 2} \theta_{0}^{2}$ for $\theta_{0}<<$ 1. Removing the sector from the shell having $\mathrm{R}^{\prime}<\mathrm{r}<\mathrm{R}^{\prime}+\Delta \mathrm{r}$ increases the resistance of that shell, and thus the total resistance, by $\rho \theta_{0}{ }^{2} \Delta r / 16 \pi R^{\prime 4}$. But the volume of the segment that was removed, $\Delta V=\pi \theta_{0}^{2} \Delta r / 16 \pi R^{\prime 4}$. Thus, the ratio of the incremental increase in resistance to the incremental volume of the material which was removed is given by

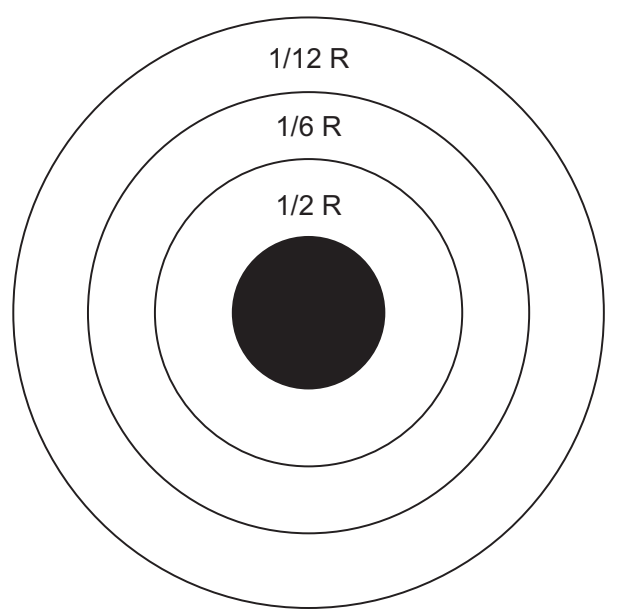

Fig. 2. Radial distribution of the resistance for the first model where $1 / 2$ of the total resistance is contained within a shell having a thickness equal to the radius of the inner sphere.

$$
\frac{\text { Change in resistance }}{\text { Volume removed }}=\frac{\rho}{16 \pi^{2} R^{\prime 4}} \text {. }
$$

That is, the sensitivity of the measured resistance to a change in the resistivity in an infinitesimal region varies inversely with the 4th power of the distance from the center of the metal sphere. For example, these measurements are 16 times more sensitive to local inhomogeneities in the resistivity at the surface of the metal sphere than to such changes at a radial distance which is equal to two times the radius of the central metal sphere.

\section{Second Model: Circular Electrode at the Surface of the Semiconductor}

It is surprising that this model is used with SSRM (Dickens, 1967) because our first model allows for the actual insertion of the probe and has a much simpler solution. However, the second model may be more suitable for SFCM because it is consistent with a current density that is normally incident at the surface of the sample. This model requires the approximation that, at the sample surface, the current is constant over a circular region with radius a and zero outside of this region. The surfaces of constant potential, which were spheres in the first model, are now oblate spheroids which are bisected by the surface of the semiconductor as shown in Fig. 3. On the $z$-axis $(\eta=1)$ the potential and the electric field are given by Eqs. (6) and (7) where $V_{0}$ is the potential of the contact relative to zero at infinite $\xi$ (Dickens, 1967).

$$
\begin{aligned}
& V=V_{0}\left[1+\frac{2}{\pi} \operatorname{Tan}^{-1}\left(\frac{z}{a}\right)\right] \\
& E_{z}=\frac{-2 V_{0}}{\pi a\left(1+\frac{z^{2}}{a^{2}}\right)}
\end{aligned}
$$

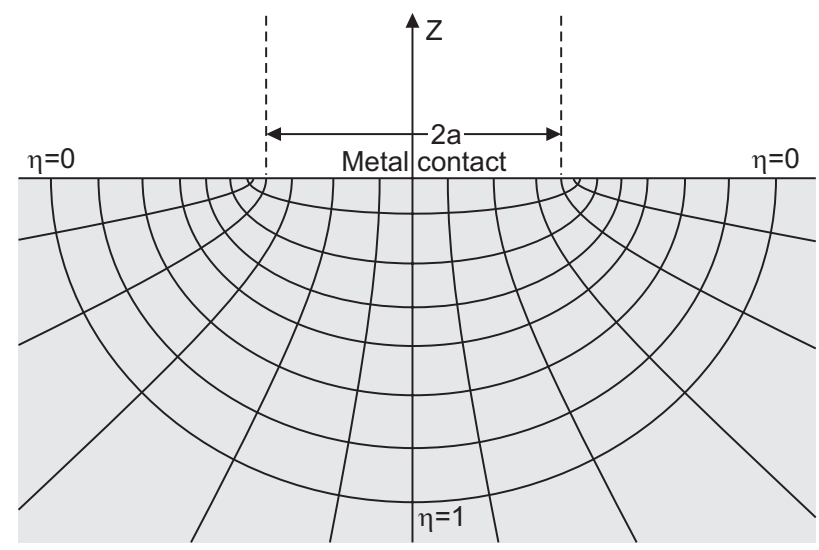

Fig. 3. Constant potential surfaces for the second model with a circular metal contact at the surface of the semiconductor where $\eta=0$. In oblate spheroidal coordinates $(\xi, \eta, \phi)$ the surfaces of constant potential have constant $\xi$. 
For large values of $\xi$ in three-dimensions the surfaces of constant potential approach the concentric spheres as seen in the first model, and on the $z$-axis for $|z|>>a$, Eq. (7) shows that $\mathrm{E}_{\mathrm{z}}$ approaches $-2 \mathrm{~V}_{0} \mathrm{a} / \pi \mathrm{z}^{2}$ which is consistent with $\mathrm{E}_{\mathrm{r}}=2 \mathrm{~V}_{0} \mathrm{a} / \pi \mathrm{r}^{2}$ in the first model.

The total spreading resistance in this model is given by Eq. (8) (Dickens, 1967).

$$
R_{S}=\frac{\rho}{4 a}
$$

Thus, the total current is given by $\mathrm{I}_{0}=4 \mathrm{aV} \mathrm{V}_{0} \rho$.

We consider this model as a series circuit of incremental resistors, each being an oblate spheroidal shell with thickness $\Delta \xi$ so the separation is tapered with a maximum spacing of $\Delta \mathrm{z}=\mathrm{a} \Delta \xi$ at the $\mathrm{z}$-axis where $\eta=1$ and $\mathrm{z}=\mathrm{z}_{0}$. Thus, from Eq. (7), the potential across each shell is given by Eq. (9) and the resistance for each shell is given by Eq. (10).

$$
\begin{gathered}
V_{S H}=\frac{2 V_{0} \Delta z}{\pi a\left(1+\frac{z_{0}^{2}}{a^{2}}\right)} \\
R_{S H}=\frac{\rho \Delta z}{2 \pi a^{2}\left(1+\frac{z_{0}^{2}}{a^{2}}\right)}
\end{gathered}
$$

Consider a tubular surface within the semiconductor having $\eta=1-\delta$ where the constant $\delta$ is much less than 1 . This tube is normal to the surfaces for constant potential, which have specific values of $\xi$, so a fixed current $\Delta \mathrm{I}$ flows through the full length of this tube. Consider the intersection of this tube with the oblate spheroid with the surface $\xi=\xi_{0}$ which is at a constant potential. Eq. (11) uses the scale factors to determine an increment in the area of this intersection:

$$
d S_{\xi}=h_{\eta} h_{\phi} d \eta d \phi=a^{2} \sqrt{\left(\xi_{0}^{2}+\eta^{2}\right)\left(\xi_{0}^{2}+1\right)} d \eta d \phi .
$$

Integrating over $\phi$ from 0 to $2 \pi$, and over $\eta$ from $A$ to $B$ gives the following expression for the area over this intersection:

$$
S_{A B}=\pi a^{2} \sqrt{\xi_{0}^{2}+1}\left[\eta \sqrt{\eta^{2}+1}+\ln \left(\eta+\sqrt{\eta^{2}+1}\right)\right]_{A}^{B} .
$$

Thus, integrating over $\eta$ from -1 to +1 gives the following expression for the full area of the surface of the oblate spheroid:

$$
S_{A B}=\pi a^{2} \sqrt{\xi_{0}^{2}+1}\left[2 \sqrt{2}+\ln \left(\frac{\sqrt{2}+1}{\sqrt{2}-1}\right)\right] .
$$

Integrating over the intersection, with $\eta$ from -1 to $-1+\delta$, gives the following expression for the area of the intersection of the tube with the oblate spheroid:

$$
\Delta S=2 \sqrt{2} \pi a^{2} \sqrt{\xi_{0}^{2}+1} \delta .
$$

Thus, the fraction of the area of the surface of the oblate spheroid that is covered by this intersection is given by the following expression:

$$
\frac{\Delta S}{S_{A B}}=\frac{2 \sqrt{2} \delta}{2 \sqrt{2}+\ln \left(\frac{\sqrt{2}+1}{\sqrt{2}-1}\right)}
$$

Following an approach similar to that which was used with the first model, we note that removing a segment of the semiconductor that is located on the $\mathrm{z}$-axis from a shell having a constant thickness of $\mathrm{a} \Delta \xi$ will increase the resistance of that shell. Note that $\Delta z$ is approximately a $\Delta \xi$ at the location of this segment near to the $\mathrm{z}$-axis. Thus, the volume of this segment is given by the following expression:

$$
\Delta V=2 \sqrt{2} \pi a^{3} \sqrt{\xi_{0}^{2}+1} \delta(\Delta \xi)
$$

Removing the segment of the semiconductor would increase the resistance of the shell by a factor of $S_{A B} /\left(S_{A B}-\Delta S\right)$. Thus, the total resistance will be increased by

$$
\begin{aligned}
& \Delta R_{S H}=\frac{R_{S H}}{1-\frac{\Delta S}{S_{A B}}}-R_{S H}=\frac{R_{S H}}{S_{A B}} \Delta S \\
& \Delta R_{S H}=\frac{\rho \Delta z}{2 \pi a^{2}\left(1+\frac{z_{0}^{2}}{a^{2}}\right)} \frac{2 \sqrt{2} \delta}{\left[2 \sqrt{2}+\ln \left(\frac{\sqrt{2}+1}{\sqrt{2}-1}\right)\right]} .
\end{aligned}
$$

For example, on the z-axis, the ratio of the incremental increase in resistance to the incremental volume of material that was removed is given by

$$
\frac{\text { Change in resistance }}{\text { Volume removed }}=\frac{1}{\left[1+\frac{1}{2 \sqrt{2}} \ln \left(\frac{\sqrt{2}+1}{\sqrt{2}-1}\right)\right]} \frac{\rho}{4 \sqrt{2} \pi^{2} a\left(a^{2}+z_{0}^{2}\right)^{\frac{3}{2}}}
$$

Eq. (19) shows that, on the z-axis, the sensitivity of the measured resistance to a change in the resistivity in an infinitesimal region of the semiconductor varies inversely with the 3 rd power of the distance from the circular electrode when this distance is much greater than the radius of the circular contact at the surface.

We have not considered high-frequency effects in this analysis so the dimensions must be much smaller than the wavelength in applications to SFCM. A first approximation for an extension to higher frequencies was described by Dickens (1967). 


\section{Mesoscopic Effects on the Electrical Spreading Resistance}

Macroscopically, an electric field $\mathrm{E}_{\mathrm{z}}$ causes a current density $\mathrm{J}_{z}$ which is equal to $\sigma \mathrm{E}_{z}$ in a medium with uniform conductivity $\sigma$. However, the apparent regularity of this current is the result of many random collisions of the carriers at a much finer level. The mean-free path $\lambda$ is defined as the mean distance traveled by a carrier between consecutive collisions (Sondheimer, 1952). Mesoscopic effects occur at a length scale which is between that for bulk matter and the sizes of individual atoms or molecules. The measured electrical resistance deviates from the classical value when the dimensions are comparable with the mean-free path for the carriers, and is quantized at much smaller dimensions comparable to atoms or molecules (Durkan, 2014).

In 1959, Little (1959) derived an expression for the phonon resistance in the Knudsen limit of heat transfer where the radius of the orifice is much smaller than the mean-free path for phonons. Wexler (1966) showed that Little's expression for the phonon resistance requires the following expression for the electrical spreading resistance:

$$
R_{S K}=\frac{4 \rho \lambda}{3 \pi a^{2}} .
$$

Independently, six years after Little, Sharvin (1965) derived an expression equivalent to Eq. (20) and now this quantity is generally referred to as the "Sharvin resistance".

The simple addition of the Sharvin resistance to the Drude resistance, as shown in Eq. (21) differs by less than 2.5\% from the exact calculation, from the Drude limit through the Sharvin limit (de Jong, 1994).

$$
R_{S D}=\frac{\rho}{4 a}+\frac{4 \rho \lambda}{3 \pi a^{2}}
$$

The mesoscopic increase in the resistance of the aperture, which is associated with ballistic transfer, may be understood from classical considerations. This effect occurs because, in ballistic transfer the transmission is proportional to the cosine of the angle of incidence because of the reduction in the intercepted area of the aperture. A tunneling junction is one type of a nanoscale aperture and this type of angular dependence is seen in plots of the modulus of the wavefunction for a tunneling junction (e.g., Fig. 2 of Avotina et al., 2005). This result suggests that the mesoscopic effects for small apertures would cause a greater fraction of the current to be present on the axis than we have shown in Model 1 and Model 2 where diffusive transport is assumed. This effect would increase the sensitivity to inhomogeneities in the carrier concentration of a semiconductor at greater distances from the surface.

\section{RESULTS}

Model 1 requires spherical symmetry, and in the limit as the aperture radius approaches zero, or equivalently at points within the semiconductor that are close to the aperture, the solution for Model 2 has a current density that is also radially symmetric within the semiconductor. However, mesoscopic effects would increase the sensitivity of the measurements to inhomogeneities at greater distances from the surface in both models. This enhancement would be more pronounced with SFCM because of the much smaller spot-size.

Eq. (5) shows that for Model 1 the sensitivity of the resistance measurement to a change in the resistivity in an infinitesimal region varies inversely with the distance 4 th power of the distance from the center of the metal sphere. However, Eq. (19) shows that for Model 2 this sensitivity varies inversely as the 4 th power of the radius of the circular contact at points close to the surface, and inversely as the 3rd power of the distance from the surface when that distance is much greater than the radius of the circular contact.

\section{DISCUSSION AND CONCLUSIONS}

In SSRM the resolution for carrier profiling is defined as the smallest lateral displacement for the probe that will provide a significant change in the measured resistance. Thus, SSRM can detect carrier depletion at dislocations in the lattice of a semiconductor (Yokoyama et al., 2009) or other variations occurring over distances that are much greater than the size of the probe. However, now we address the sensitivity of SSRM and SFCM measurements to heterogeneities at specific distances and directions relative to the point where the measurements are made. This is a first step toward a technique for determining the carrier profile with much greater resolution by combining the measurements made at multiple locations. We anticipate that such a technique may be more successful with SFCM because of the much finer "spotsize" and the nondestructive nature of the measurement.

It appears that Model 1 would be more appropriate with SSRM when the probe penetrates the sample whereas Model 2 would be more appropriate with SFCM when a normal current is incident on the surface of the semiconductor.

When considering mesoscopic effects, it is necessary for the carriers to have a path length several times greater than their mean-free path in order to have sufficient scattering to reach equilibrium with the local properties of a semiconductor. Several recent papers have described SSRM measurements with silicon samples having dopant concentrations for which the mean-free path of the carriers is from 2.3 to 15 nm (Hantschel et al., 2009, 2015, 2016). Thus, it appears that nanometer or sub-nanometer resolution would not be possible with these samples. 


\section{CONFLICT OF INTEREST}

We do not consider that there is a conflict of interest because, while some of the phenomena for Scanning Frequency Comb Microscopy have been demonstrated, there is no commercial product at the present time.

\section{ACKNOWLEDGMENTS}

This work was performed in part, at the Center for Integrated Nanotechnologies, a U.S. Department of Energy, Office of
Basic Energy Sciences user facility. Los Alamos National Laboratory, an affirmative action equal opportunity employer, is operated by the Los Alamos National Security, L.L.C., for the National Nuclear Security Administration of the U.S. Department of Energy under Contract No. DE-AC52-06NA25396. We acknowledge support from the U.S. Department of Energy under Award No. DESC0006339 when the Microwave Frequency Comb was first demonstrated. This work is currently supported by the National Science Foundation under Grant 1648811.

\section{REFERENCES}

Avotina Ye S, Kolesnichenko Yu A, Omelyanchouk A N, Otte A F, and Van Ruintenbeek J M (2005) Method to determine defect positions below a metal surface by STM. Phys. Rev. B 71, 115430.

Chien A A and Karamcheti V (2013) Moore's Law: the first ending and a new beginning. Computer (IEEE) 46, 48-53.

de Jong M J M (1994) Transition from Sharvin to Drude resistance in high-mobility wires. Phys. Rev. B 49, 7778-7781.

Dickens L E (1967) Spreading resistance as a function of frequency. IEEE Trans. Microwave Theory Tech. 15, 101-109.

Dunham S T, Collins N, and Jeng N (1994) Improved analysis of spreading resistance measurements. J. Vac. Sci. Technol. B 12, 283-289.

Durkan C (2014) Current at the Nanoscale (World Scientific, New Jersey).

Hagmann M J, Andrei P, Pandey S, and Nahata A (2015) Possible applications of scanning frequency comb microscopy for carrier profiling in semiconductors. J. Vac. Sci. Technol. B 33, $02 B 109$.

Hagmann M J and Henage T E (2016) Simplified calculations of the lateral distribution for the current in tunneling junctions having general shapes. Electron. Lett. 52, 395-396.

Hagmann M J, Taylor A J, and Yarotski D A (2012) Observation of 200th harmonic with fractional linewidth of $10^{-10}$ in a microwave frequency comb generated in a tunneling junction. Appl. Phys. Lett. 101, 241102.

Hagmann M J, Yarotski D A, and Mousa M S (2017) Microwave frequency comb from a semiconductor in a scanning tunneling microscope. Microsc. Microanal. 23, 443-448.

Hantschel T, Demeulemeester C, Eyben P, Schulz V, Richard O, Bender $\mathrm{H}$, and Vandervorst W (2009) Conductive diamond tips with sub-nanometer electrical resolution for characterization of nanoelectronics device structures. Phys. Stat. Solid. A 206, 2077 2081.
Hantschel T, Tsigkourakos M, Kluge J, Werner T, Zha L, Paredis K, Eyben P, Nuytten T, Xu Z, and Vandersorst W (2015) Overcoated diamond tips for nanometer scale semiconductor device characterization. Microelectron. Eng. 141, 1-5.

Hantschel T, Tsigkourakos M, Zha L, Nuytten T, Paredis K, Majeed B, and Vandervorst W (2016) Diamond scanning probes with sub-nanometer resolution for advanced nanoelectronics device characterization. Microelectron. Eng. 159, 46-50.

Kelly T F and Miller M K (2007) Invited review article: atom probe tomography. Rev. Sci. Instrum. 78, 031101.

Little W A (1959) The transport of heat between dissimilar solids at low temperatures. Can. J. Phys. 37, 334-349.

Mack C A (2011) Fifty years of Moore's Law. IEEE Trans. Semicond. Manuf. 24, 202-207.

Rathod G B, Paul R P, Solanki M K M, Patel H J, and Bareja M N (2014) Review on extreme ultraviolet lithography. Int. J. Adv. Res. Comput. Sci. Softw. Eng. 4, 682-687.

Sharvin Y V (1965) A possible method for studying fermi surfaces. Sov. Phys. JETP 21, 655-656.

Sondheimer E H (1952) The mean free path of electrons in metals. Adv. Phys. 1, 1-42.

Vandervorst W, Schulze A, Kambham A K, Mody J, Gilbert M, and Eyben P (2014) Dopant/carrier profiling for 3D-structures. Physica Status Solidi C 11, 121-129.

Wexler G (1966) The size effect and the non-local Boltzmann transport equation in orifice and disk geometry. Proc. Phy. Soc. London $\mathbf{8 9}$, 927-941.

Yokoyama T, Takenaka R, Kamimura Y, Edagawa K, and Yonenaga I (2009) Direct observation of carrier depletion around a dislocation in GaP by scanning spreading resistance microscopy. Appl. Phys. Lett. 95, 202108. 